\title{
Immigration can destabilize tri-trophic interactions: implications for conservation of top predators
}

\author{
Kevin L. S. Drury • Jesse D. Suter • Jacob B. Rendall • \\ Andrew M. Kramer · John M. Drake
}

Received: 20 February 2014 / Accepted: 26 December 2014 / Published online: 14 January 2015

(c) Springer Science+Business Media Dordrecht 2015

\begin{abstract}
Top predators often have large home ranges and thus are especially vulnerable to habitat loss and fragmentation. Increasing connectance among habitat patches is therefore a common conservation strategy, based in part on models showing that increased migration between subpopulations can reduce vulnerability arising from population isolation. Although three-dimensional models are appropriate for exploring consequences to top predators, the effects of immigration on tri-trophic interactions have rarely been considered. To explore the effects of immigration on the equilibrium abundances of top predators, we studied the effects of immigration in the three-dimensional Rosenzweig-MacArthur model. To investigate the stability of the top predator equilibrium, we used MATCONT to perform a bifurcation analysis. For some combinations of model parameters with low rates of top predator immigration, population trajectories spiral towards a stable focus. Holding other parameters constant, as immigration rate is increased, a supercritical Hopf bifurcation results in a stable limit cycle and thus top predator populations that cycle between high and low abundances. Furthermore, bistability arises as immigration of the intermediate predator is increased. In this case, top predators may exist at relatively low abundances while prey become extinct, or for other initial conditions, the relatively higher top predator abundance controls intermediate predators allowing for non-zero prey population abundance and increased diversity. Thus,
\end{abstract}

K. L. S. Drury $(\bowtie) \cdot$ J. D. Suter · J. B. Rendall

Department of Mathematical and Engineering Sciences,

Bethel College, Mishawaka, IN, USA

e-mail: kevin.drury@ bethelcollege.edu

A. M. Kramer · J. M. Drake

Odum School of Ecology, University of Georgia,

Georgia, USA our results reveal one of two outcomes when immigration is added to the model. First, over some range of top predator immigration rates, population abundance cycles between high and low values, making extinction from the trough of such cycles more likely than otherwise. Second, for relatively higher intermediate predator migration rates, top predators may exist at low values in a truncated system with impoverished diversity, again with extinction more likely.

Keywords Tri-trophic interactions - Three-dimensional Rosenzweig-MacArthur model · Bifurcation · Supercritical Hopf bifurcation · Bistability · MATCONT · Predator conservation

\section{Introduction}

Top ecosystem predators typically have expansive habitat requirements (Holt 1993) making them especially vulnerable to habitat loss. Loss of such predators is a major conservation concern due to their unique functional roles, cascading effects on community structure, control of species' abundance at lower trophic levels, and hence maintenance of biodiversity (Sergio et al. 2006). Top predators also influence the behavior of individuals in trophic levels beneath them, an effect that often propagates to ecosystem-level consequences (Beschta 2003; Ripple and Beschta 2004; Heithaus et al. 2007). Thus, habitat modifications with concomitant decreases in top predator numbers is one of the key conservation issues of recent decades. Examples of once-common North American top predators that are now rare include grizzly bears (Ursus arctos horribilis) because of habitat loss (Wielgus 2002), lynx (Lynx canadensis) because of habitat fragmentation (Koehler et al. 2008), and cougars (Puma concolor) because of hunting and exploitation (Robinson et al. 2008). 
Isolation of subpopulations is implicated in local extinctions (Diamond 1984; Holyoak and Lawler 1996; Hill et al. 1996), and thus, connectivity between subpopulations is often suggested as a way to stabilize populations of such top predators (Rodriguez and Delibes 2003; Miotto et al. 2011). This connectivity is a way of increasing effective patch size, which is important, because in general, extinction rates of many organisms are inversely correlated with patch size (Holt 1993). Even though the community- and ecosystem-level effects of predators are mediated by cascading trophic relations, the effects of predator immigration on communities of three interacting species have rarely been considered. We use a mathematical model to show that, counter-intuitively, increased immigration can both destabilize top predator population dynamics and generate bistability. In both cases, the potential for top predators to tend to low densities is increased. Thus, when tri-trophic species interactions are taken into consideration, it appears that immigration itself may actually further endanger populations of large predators.

These results are counter-intuitive because both spatially implicit (e.g., Hanski 1994) and spatially explicit (e.g., Bascompte and Sole 1996) mathematical models predict population persistence to be enhanced by immigration. Indeed, immigration stabilizes otherwise chaotic onedimensional difference equation models, such as the Ricker model (Holt et al. 1983; McCallum 1992; Stone 1993), although it destabilizes the discrete logistic map (Holt et al. 1983), and can either stabilize, or destabilize, simple twospecies continuous-time systems (Holt 2002). To illustrate stabilization, we add immigration to the predator equation of the classic two-dimensional Rosenzweig-MacArthur model and consider the effect of increasing the migration parameter $\mu$ from zero. With predator immigration $\mu \geq 0$, the model is

$$
\begin{aligned}
& \frac{d x}{d t}=x(1-x)-\frac{\alpha x y}{1+\beta x} \\
& \frac{d y}{d t}=\mu+\frac{\alpha x y}{1+\beta x}-\delta y
\end{aligned}
$$

where $\alpha$ is the maximum attack rate of predators on prey, $\beta$ is the prey density at which predator attack rate is one half the maximum, and $\delta$ is the predator mortality rate. Figure 1 shows that as migration of the predator varies, dynamics change qualitatively. The dotted lines represent stable oscillations and are generated with migration rate $\mu=0.0$. The resulting limit cycle surrounds an unstable focus, for which the Jacobian matrix has a complex conjugate pair of eigenvalues with positive real part. First, increasing predator immigration slightly to $\mu=0.25$ generates a stable focus represented by the dashed curve (i.e., complex conjugate eigenvalues with negative real part) and second, increasing predator immigration further to $\mu=1.25$ results in a stable node (i.e., real, negative eigenvalues), drawn as a solid curve culminating at the asterisk. This simple example illustrates why, in practice, migration corridors have been advocated to allow migration between subpopulations (reviewed in Holt (1993), pp. 82-83).

Instability, nevertheless, arises because of additional nonlinearities in the population interaction terms when an equation is added for a top predator. Indeed, evidence from some empirical systems reveals that population cycles are likely generated through three-trophic-level interactions (Krebs et al. 1995). We found such oscillations in our bifurcation analysis of the three-dimensional Rosenzweig-MacArthur model. This suggests that in the presence of some
Fig. 1 Changes in dynamics of the modified two-dimensional Rosenzweig-MacArthur model as predator immigration rate $\mu$ is varied. For $\mu=0$ (dotted curve), predator and prey oscillate in a limit cycle (i.e., eigenvalues are $0.04+0.23 i, 0.04-0.23 i)$. As $\mu$ is increased to 0.25 , these oscillations die out exponentially resulting in a stable focus (dashed curve) and the system has eigenvalues of $-0.07+0.8 \mathrm{i},-0.07-0.8 \mathrm{i}$. Finally, for $\mu=1.25$, predator and prey populations move to equilibrium abundances at a stable node (the asterisk approached by the solid curve) with eigenvalues $-0.68,-0.27$

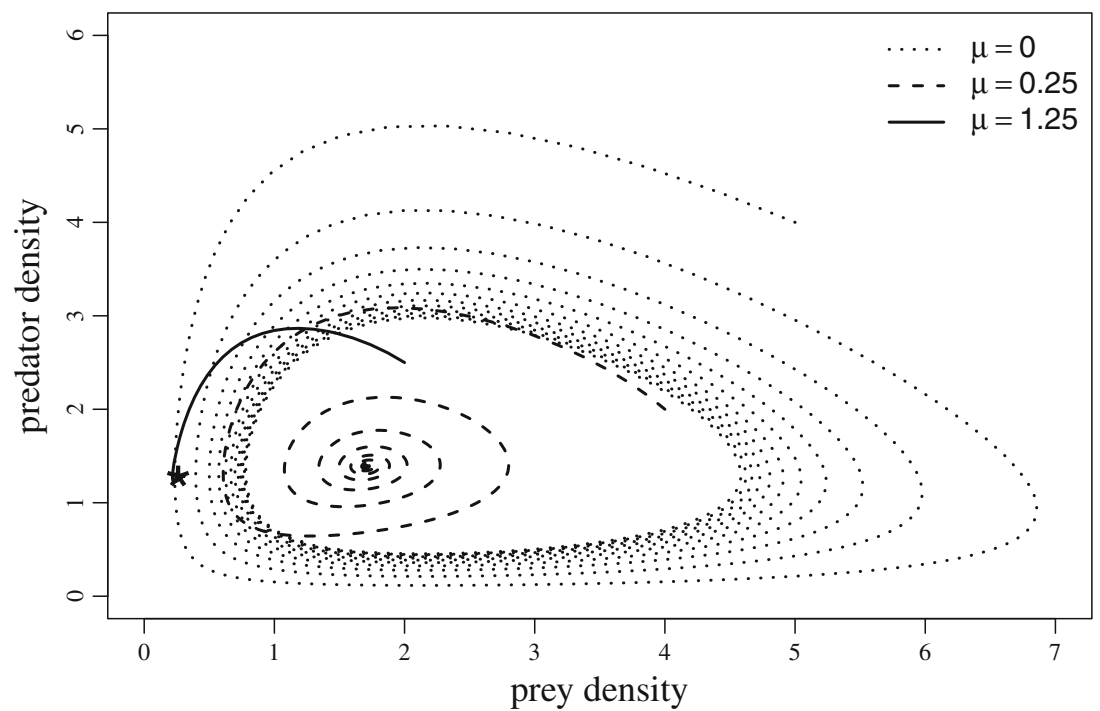


levels of immigration, top predator population abundance may cycle and therefore periodically visit relatively low densities. Such unexpected results, including such phenomena as the paradox of enrichment (Rosenzweig 1971), frequently arise in nonlinear dynamics. Indeed, other studies have similarly found immigration to sometimes be destabilizing (Holt 1985; Kuang and Takeuchi 1994). Thus, to the extent that model dynamics represent those of real ecosystems, our results suggest that practical strategies for enhancing inter-patch migration should be informed by modeling studies that explore suitable migration rates to avoid introduction of destabilizing oscillations.

\section{The classic three-dimensional Rosenzweig-MacArthur model}

In fully dimensional form, the three-dimensional Rosenzweig-MacArthur model is,

$$
\begin{aligned}
& \frac{d X}{d T}=R X(1-X / K)-\frac{A_{x} X Y}{B_{x}+X}, \\
& \frac{d Y}{d T}=\frac{C_{x, y} A_{x} X Y}{B_{x}+X}-\frac{A_{y} Y Z}{B_{y}+Y}-\delta_{y} Y, \\
& \frac{d Z}{d T}=\frac{C_{y, z} A_{y} Y Z}{B_{y}+Y}-\delta_{z} Z,
\end{aligned}
$$

where $X$ represents the resource (prey), $Y$ represents the primary consumer, often called the predator or intermediate predator, and $Z$ represents a secondary consumer, our "top predator." The parameters in Eqs. 3-5 are described in Table 1.

Table 1 Parameters of the fully dimensional three-dimensional Rosenzweig-MacArthur model and their descriptions

\begin{tabular}{ll}
\hline Parameter & Description \\
\hline$R$ & Prey population growth rate \\
$K$ & Prey carrying capacity \\
$A_{x}$ & Maximal attack rate of $Y$ on $X$ \\
$B_{x}$ & Prey density at which $Y$ is half saturated \\
$C_{x, y}$ & Factor to convert $X$ to $Y$ \\
$A_{y}$ & Maximal attack rate of $Z$ on $Y$ \\
$B_{y}$ & $Y$ density at which $Z$ is half saturated \\
$\delta_{y}$ & Non-predation mortality of $Y$ \\
$C_{y, z}$ & Factor to convert $Y$ to $Z$ \\
$\delta_{z}$ & Mortality of $Z$ \\
\hline
\end{tabular}

Comparison to the two-dimensional Rosenzweig-MacArthur model

Equations 3-5 have been analyzed extensively, but analyses use different non-dimensionalization and reparameterization schemes, resulting in different final forms of the model. Perhaps the most common scheme scales out $R$ and $K$ (e.g., Hastings and Powell 1991; Kuznetsov and Rinaldi 1996). We wish to facilitate comparison of a classic feature of the more familiar two-dimensional RosenzweigMacArthur model, however, and so we instead follow (Kuznetsov et al. 2001) and intentionally retain these (initially). Then, after our comparison, we pursue a different parameterization.

Some model simplification allows us to take advantage of published parameter values (see, e.g., Kuznetsov et al. 2001) and so we begin by factoring $B_{x}$ and $B_{y}$ from the denominators of the functional responses and then renaming $\frac{1}{B_{x}} \equiv b_{x}$ and $\frac{1}{B_{y}} \equiv b_{y}$. Finally, we rename $\frac{A_{x}}{B_{x}} \equiv a_{x}$ and $\frac{A_{y}}{B_{y}} \equiv a_{y}$ yielding,

$\frac{d X}{d T}=R X(1-X / K)-\frac{a_{x} X Y}{1+b_{x} X}$,

$\frac{d Y}{d T}=\frac{C_{x, y} a_{x} X Y}{1+b_{x} X}-\delta_{y} Y-\frac{a_{y} Y Z}{1+b_{y} Y}$,

$\frac{d Z}{d T}=\frac{C_{y, z} a_{y} Y Z}{1+b_{y} Y}-\delta_{z} Z$.

We used the parameter values in Table 2 and a numerical continuation algorithm (described later) to solve for the equilibria of Eqs. 6-8 over a range of values of $K$ to generate Fig. 2. The $y$-axis values are equilibria of the $X, Y$, and $Z$ populations, respectively. For small values of $K$ (the left end of the $x$-axis), only the prey population can invade the system; predators and top predators are stable at abundance zero. After the transcritical bifurcation at $T C_{y}$, intermediate predators can invade the system and all increases in $K$ are translated into predator biomass; prey abundance remains constant. After the second transcritical

Table 2 Parameters values used in Eqs. 6-8 to generate Fig. 2

\begin{tabular}{llll}
\hline Parameter & Value & Parameter & Value \\
\hline$R$ & 1 & $\delta_{y}$ & 0.3 \\
$a_{x}$ & 0.09 & $a_{y}$ & 0.1 \\
$b_{x}$ & 0.1 & $b_{y}$ & 0.2 \\
$C_{x, y}$ & 1 & $C_{y, z}$ & 1 \\
$K$ & Varies & $\delta_{z}$ & 0.29 \\
\hline
\end{tabular}




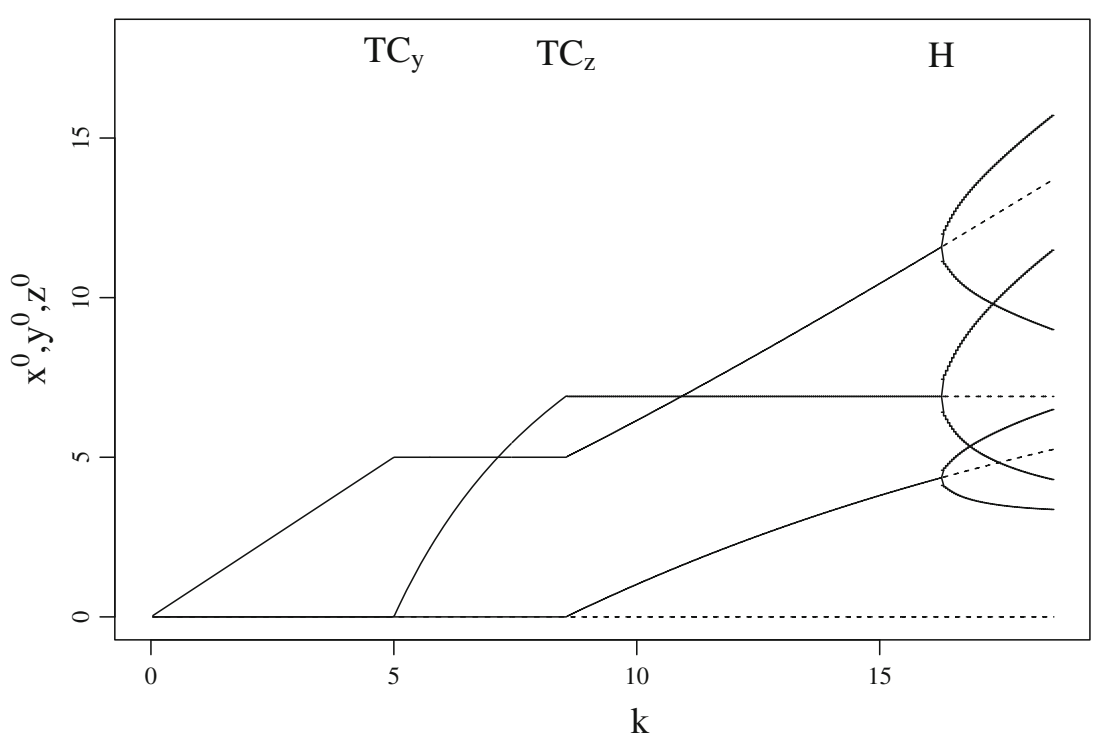

Fig. 2 Solution diagram for Eqs. 6-8 demonstrating the paradox of enrichment in the three-dimensional Rosenzweig-MacArthur model. $X^{0}, Y^{0}, Z^{0}$ represent the equilibria of $X, Y$, and $Z$, respectively. For the interval $0<k<T C_{y}$, only prey can invade the system. Between $T C_{y}<k<T C_{z}$, the predator invades the system and all increases in $k$ are translated into predator biomass. Between $T C_{z}<k<H$, the top

bifurcation at $T C_{z}$, however, top predators invade the system and increase along with prey as $K$ is increased further, while predator abundance remains constant. A supercritical Hopf bifurcation occurs at point $H$ whereby the stable focus loses stability (represented by the dashed curves) and a limit cycle appears (limit cycle minima and maxima represented by solid lines surrounding the dashed curves).

This is a three-dimensional version of the classic paradox of enrichment, typically shown in the two dimensions of prey and predator. The paradox is that enriching the environment (increasing the carrying capacity of the prey in the absence of predator regulation) can lead to low population abundances at the low points of cycles. Thus, we see that nonlinear feedbacks from the two-dimensional case persist in the three-dimensional model. Our key result, namely that immigration can destabilize dynamics for a given value of $K$, demonstrates that other similar counter-intuitive results (e.g., Doebeli 1995) emerge after the addition of the third nonlinear equation. Indeed, in still higher dimensions even more possibilities exist. For example, Boer et al. (2001) found that top predators in a four-dimensional chemostat model may even go extinct as the environment is increasingly enriched.

The conventional reparameterization

Having compared the two- and three-dimensional models, we return to Eqs. 3-5 and consider a more predator invades the system and both prey and top predator increase in biomass while the intermediate predator remains constant. At the point $H$, a supercritical Hopf bifurcation occurs leading to stable limit cycles for all species. The paradox is that enriching the environment such that it can support more prey destabilizes the populations generating low population abundances at the low point of the limit cycle

conventional non-dimensionalization of the threedimensional model. The non-dimensionalization is reproduced in detail in Appendix A and the resulting equations are,

$$
\begin{aligned}
& \dot{x}=x(1-x)-\frac{\alpha_{x} x y}{1+\beta_{x} x}, \\
& \dot{y}=\frac{\alpha_{x} x y}{1+\beta_{x} x}-\delta_{y} y-\frac{\alpha_{y} y z}{1+\beta_{y} y}, \\
& \dot{z}=\frac{\alpha_{y} y z}{1+\beta_{y} y}-\delta_{z} z .
\end{aligned}
$$

This model has a long and distinguished history in generating insights into tri-trophic ecosystem dynamics. Additionally, these have become the workhorse equations for understanding dynamics of three-dimensional nonlinear systems (Hastings and Powell 1991; Kuznetsov and Rinaldi 1996; van Voorn et al. 2010). We therefore use this model as our base model to which we then add immigration.

The three-dimensional Rosenzweig-MacArthur model with constant immigration

We modified the system of Eqs. 9-11 to include $\mu_{x}, \mu_{y}$, and $\mu_{z}$ representing constant immigration of prey, predators, 
and top predators, respectively, generating the system of equations,

$\dot{x}=\mu_{x}+x(1-x)-\frac{\alpha_{x} x y}{1+\beta_{x} x}$,

$\dot{y}=\mu_{y}+\frac{\alpha_{x} x y}{1+\beta_{x} x}-\delta_{y} y-\frac{\alpha_{y} y z}{1+\beta_{y} y}$,

$\dot{z}=\mu_{z}+\frac{\alpha_{y} y z}{1+\beta_{y} y}-\delta_{z} z$

Our approach therefore follows Holt's study of the twodimensional Rosenzweig-MacArthur model (Holt 2002). Including migration in such models corresponds to modeling what Amarasekare (2008) calls extinction-immigration dynamics, where the state variable is species abundance as opposed to patch occupancy patterns. For the purposes of the first part of this study, we set $\mu_{x}=0$ and $\mu_{y}=0.01$ and varied $\mu_{z}$. This corresponds to stationary prey and relatively less-mobile predators, with varying mobility of top predators $\left(\mu_{z}\right)$ to simulate more or less connectivity between habitat patches. Such variability in mobility across species and trophic levels is widely accepted (Wiens 1989).

\section{Continuing equilibria with MATCONT}

The equilibria of Eqs. 9-10 are well known (e.g., van Voorn et. al 2010) and are listed in Table 3.

The final equilibrium in Table 3 is,

$$
\begin{gathered}
x^{*}=\frac{\beta_{x}-1+\sqrt{\left(\beta_{x}+1\right)^{2}-\frac{4 \alpha_{x} \beta_{x} \delta_{z}}{\alpha_{y}-\beta_{y} \delta_{z}}}}{2 \beta_{x}}, \\
y^{*}=\frac{\delta_{z}}{\alpha_{y}-\beta_{y} \delta_{z}}, \\
z^{*}=\frac{f\left(x^{*}\right)-\delta_{x}}{\alpha_{y}-\beta_{y} \delta_{z}},
\end{gathered}
$$

with

$f(x)=\frac{\alpha_{x} x}{1+\beta_{x} x}$.

Numerous software packages exist that allow one to compute such equilibria numerically, then vary a focal

Table 3 Equilibria of the non-dimensionalized three-dimensional Rosenzweig-MacArthur model and their descriptions

\begin{tabular}{ll}
\hline Equilibrium & Description \\
\hline$(0,0,0)$ & Trivial equilibrium \\
$(1,0,0)$ & Prey-only equilibrium \\
$\left(\frac{\delta_{y}}{\alpha_{x}-\delta_{y} \beta_{x}},\left(1-x^{*}\right)\left(1+\beta_{x} x^{*}\right) / \alpha_{x}, 0\right)$ & Prey and intermediate \\
$\left(x^{*}, y^{*}, z^{*}\right)$ & predator equilibrium \\
\hline
\end{tabular}

parameter, and repeatedly recompute the equilibrium at the new parameter value. This procedure is known as "equilibrium continuation" because we "continue," i.e., continuously reevaluate, the equilibrium, in some parameter space. We used MATCONT, which is a Matlab ${ }^{\circledR}$-based continuation program to continue the equilibrium analogous to $\left(x^{*}, y^{*}, z^{*}\right)$ above, except that the equilibrium we were interested in was for Eqs. 12-14.

During the process of continuing such equilibria, special points called singularities can emerge (Govaerts et al. 2011, pg.14). Numerous test functions that have regular zeros at such singularities have been devised (see, e.g., Kuznetsov (2004), pp. 526-527 for detecting saddle-node and Hopf bifurcations). For example, at a saddle-node bifurcation point, the determinant of the system of linearized equations (i.e., the Jacobian matrix) goes to zero because one eigenvalue vanishes. Similarly, at a Hopf bifurcation point, the real part of a pair of eigenvalues passes through zero, which is captured by a test function involving the bialterate matrix product (explained in Kuznetsov (2004), pp. 528-529).

\section{Bifurcation results}

To enhance our intuition about what results to expect in our bifurcation analysis, we first added immigration to the simpler three-species model with the linear functional responses of Lotka-Volterra dynamics. Specifically,

$$
\begin{gathered}
\dot{x}=\mu_{x}+x(1-x)-\alpha_{1} x y, \\
\dot{y}=\mu_{y}+\alpha_{1} x y-\delta_{1}-\alpha_{2} y z, \\
\dot{z}=\mu_{z}+\alpha_{2} y z-\delta_{2} z .
\end{gathered}
$$

When an equilibrium of Eqs. 19-21 exists, it is known to be stable. The immigration terms in these equations do not cause bifurcations of those stable equilibria, except that immigration in higher trophic levels can cause extinction in lower trophic levels. But, what about the more complicated case of Eqs. 12-14?

Table 4 Parameter values from the literature (van Voorn et al. 2010) for the three-dimensional Rosenzweig-MacArthur model

\begin{tabular}{llll}
\hline Parameter & Value & Parameter & Value \\
\hline$\alpha_{x}$ & 5 & $\delta_{z}$ & 0.015 \\
$\beta_{x}$ & 3 & $\mu_{x}$ & 0 \\
$\delta_{y}$ & 0.25 & $\mu_{y}$ & 0.01 \\
$\alpha_{y}$ & 0.1 & $\mu_{z}$ & varies \\
$\beta_{y}$ & 2 & & \\
\hline
\end{tabular}




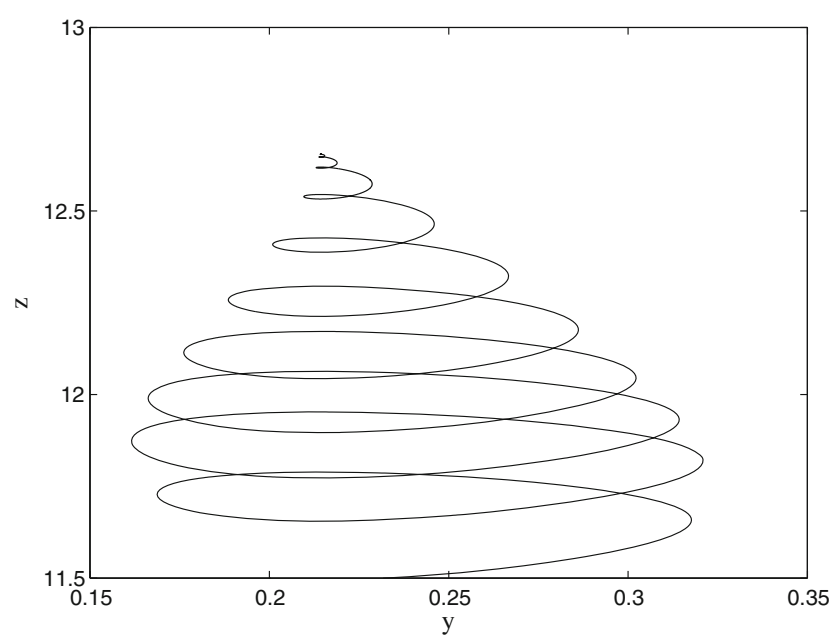

Fig. 3 The three-dimensional Rosenzweig-MacArthur model approaching a stable focus with top predator immigration $\mu_{z}=0.01$

We used published values of the parameters of Eqs. 9-11, which we reproduce in Table 4 (van Voorn et al. 2010).

We began our bifurcation analysis by seeking an equilibrium to continue. For the parameter values in Table 4, with $\mu_{z}=0.01$, the system comes to a focus (point equilibrium approached in a spiral), which we show in the $(y, z)$-plane in Fig. 3.

With initial values $\left(x_{0}, y_{0}, z_{0}\right)=(0.6,0.2,11.5)$, the system approaches the equilibrium point, in Fig. 3, namely $\left(x^{*}, y^{*}, z^{*}\right)=(0.6700,0.1986,12.7617)$. When continued (i.e., monitored as top predator immigration $\mu_{z}$ varies), this equilibrium undergoes a supercritical Hopf bifurcation as the focus loses stability and a limit cycle appears, as shown in Fig. 4. In the figure, the solid curves above and below the dashed unstable equilibrium represent the maximum and minimum values attained by the stable limit cycle.

As can be seen in the figure, as $\mu_{z}$ is increased further, the supercritical Hopf bifurcation is reversed, and this limit cycle disappears, returning the system to a stable focus. These bifurcations are plotted in the $\left(\mu_{z}, z\right)$-plane. Recall that the $y$-axis value is rescaled (i.e., amplified by a fraction of the prey carrying capacity $K$ ) and so it is not the rather minimal difference between say, 13 at the unstable equilibrium and 12 at the low point on the limit cycle that is the issue. Instead, it is the onset of cycling that is the issue. In unscaled predator units, such cycling brings the predator population abundance close to the extinction axis.

Of course, as other parameters are varied, the location in $\mu_{z}-z^{*}$ space as well as the nature of the stable limit cycle vary. For example, by simultaneously varying $\mu_{y}$, the mid-level predator immigration rate, along with $\mu_{z}$, we can trace a curve of Hopf bifurcations in the $\left(\mu_{z}, \mu_{y}\right)$ space, as in Fig. 5. Below this curve lie immigration parameter pairs that generate limit cycles (see inset) and above the curve, the immigration parameter pairs generate stable nodes (right of the hump, see inset) and stable foci (left of the hump, no inset).

Other phenomena arise for other values of the remaining parameters. For example, with non-zero $\mu_{y}=0.3$, a saddlenode bifurcation has yielded three equilibria. Two of these are unstable, however, (the dotted portions of the s-curve in Fig. 6). Nevertheless, a stable alternative to the upper stable equilibrium exists. All initial conditions below the upper dotted portion of the curve evolve towards the stable equilibrium at the relatively straight solid line. All points along this solid curve are obviously lower equilibrium values of top predator. But, these points are further distinguished by the prey being absent from the system. The two predators
Fig. 4 Top predator densities at which Hopf bifurcations occur as $\mu_{z}$ is varied

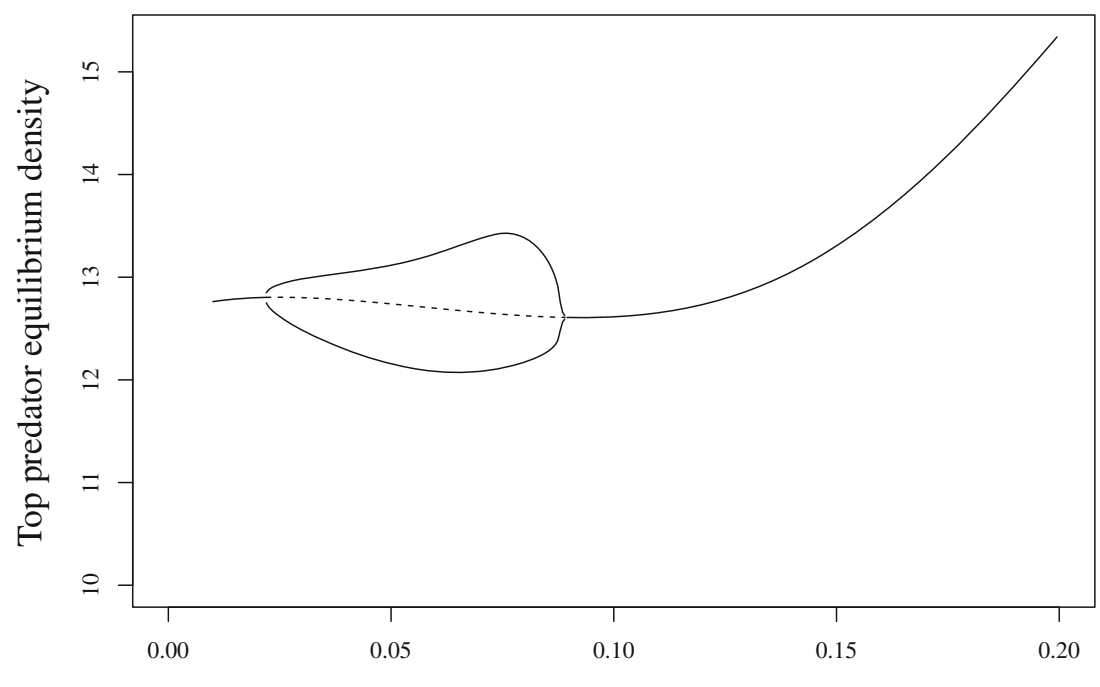

Top predator immigration 
Fig. 5 Curve of Hopf bifurcations generated by varying $\mu_{z}$ and $\mu_{y}$ simultaneously

Fig. 6 Bistability between a predator only equilibrium (solid straight line), maintained by immigration, and a higher top predator equilibrium (solid portions of the s-curve) for which all species are present
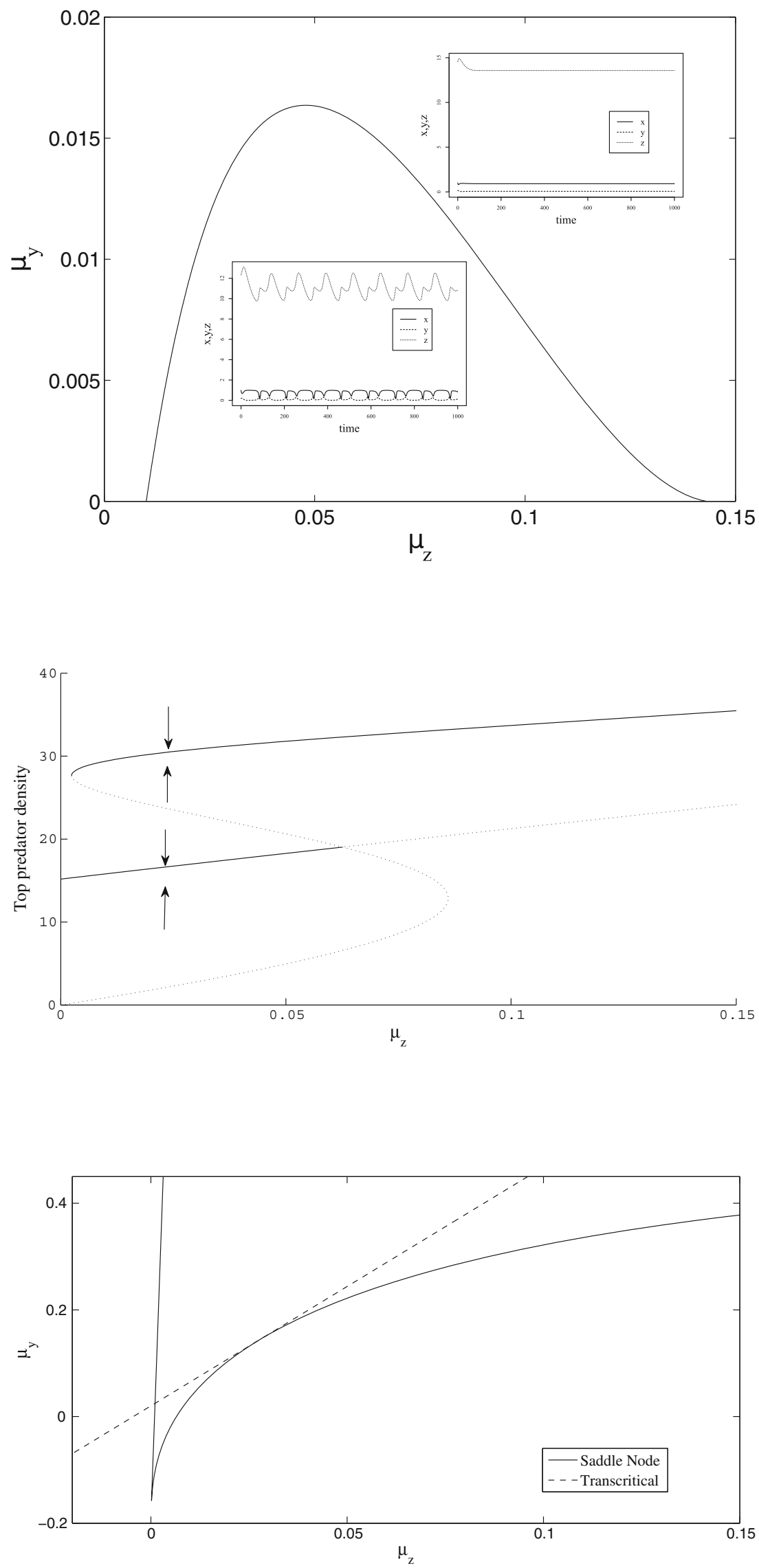

Fig. 7 Wedge of limit points of the s-shaped fold bifurcation in the two parameter space of $\mu_{y}$ and $\mu_{z}$ (solid lines). The dashed line represents parameter pairs for which the prey can invade the system 
are maintained in the system by immigration in the absence of the prey. Only for relatively high values of top predator immigration does the top predator "control" the intermediate predator to levels at which the prey can invade the system. When that happens, top predator abundance tends to the upper equilibrium. Thus, for relatively low values of top predator immigration, diversity is decreased in the system, and top predators exist at a more vulnerable equilibrium level.

In MATCONT, it is possible to continue the so-called "limit points" in Fig. 6 (i.e., the turning points of the s-curves) in a two-parameter space. The previous figure revealed that these bifurcations exist, but Fig. 7 reveals what happens to these bifurcations as parameters vary. We chose $\mu_{y}$ and $\mu_{z}$ as our bifurcation parameters and continued the bifurcation points to see how they vary as these two parameters vary (Fig. 7). Similarly, in Fig. 7, we add a dashed line representing the transcritical bifurcation whereby prey can invade the system, despite immigration rates of the intermediate predator. Notice that this occurs at relatively higher values of top predator immigration suggesting that prey can only invade the system when top predators control intermediate predators sufficiently to release the prey from predation pressure.

\section{Discussion}

Top ecosystem predators often contribute disproportionately to ecosystem functioning by, for example, enhancing diversity in trophic levels beneath them (e.g., Paine 1966). Thus, conservation efforts are often devised to enhance top predator persistence. Because such predators are typically wide ranging, with expansive habitat requirements, they are especially vulnerable to habitat loss. A staple of top predator conservation strategies has therefore been to increase effective habitat size by promoting migration between interconnected habitat patches. Although previous work has shown that this approach can stabilize inherently unstable predator and prey interactions (see, e.g., Holt 1993; Ruxton 1996), analysis of the relatively simple threedimensional Rozensweig-MacArthur model with immigration shows how easy it is for this approach to backfire (also, see Holt (2002), for a similar result in two dimensions).

\section{Migration-driven limit cycles}

For example, we have shown that for certain levels of immigration of top predators, the stable point equilibrium of the system undergoes a supercritical Hopf bifurcation and becomes unstable, while stable population cycles emerge. These cycles persist over a sizable range of immigration values, with first increasing, and then decreasing amplitude of the cycles, as immigration rate increases. As immigration levels are increased further, however, the bifurcation is reversed during which the population cycle disappears and the stable equilibrium once again gains stability. Thus, our results demonstrate that increased migration is not a panacea for population persistence. In fact, at some levels, immigration can destabilize top predator populations.

Abdllaoui et al. (2007) similarly found that limit cycles can emerge as density-dependent migration increases in two-patch predator and prey models. When considering larger numbers of patches in a lattice, Blasius et al. (1999) found that not only did populations cycle, but the populations became phase synchronized, although local peak populations were uncorrelated. Similarly, for small metacommunities following chemostat dynamics with non-random dispersal of the lowest trophic level (nutrients in their case), Suzuki and Yoshida (2012) demonstrated the conditions under which local populations became synchronized thus increasing the risk of simultaneous extinctions.

Nevertheless, the stabilizing effects of immigration that we demonstrated earlier for the two-dimensional case are also present in the three-dimensional RosenzweigMacArthur model. For example, Ruxton (1996) showed that adding immigration to the prey equation can reduce the extent to which the model exhibits chaotic behavior. Indeed, this model is well-known for generating chaotic dynamics in realistic parameter regimes (McCann and Yodzis 1996), and in fact, the entire class of models to which it belongs can generate chaos (Klebanoff and Hastings 1994). Besides being undesirable because of the possibility of abnormally low population abundances, chaotic dynamics can be transient and populations can suddenly disappear (Hastings 1996). Although McCann and Yodzis (1996) demonstrated that chaos is possible in this model for a wide variety of biologically realistic parameter combinations, those are, nevertheless, not in the range that we explored here.

\section{Migration-driven bistability}

Furthermore, when intermediate predator immigration is also increased, the possibility of bistability arises. Two stable states exist and initial conditions determine which is ultimately realized. For example, for initial conditions in the basin of attraction of the lower equilibrium, the top predator population settles on a relatively low equilibrium value. For these lower values of top predator abundance, prey are missing from the system and both predator species are maintained by immigration. Thus, both intermediate and top predators exist in sink populations for this case. Only once top predator immigration exceeds a threshold value can the prey invade the system because top predator abundance is then adequate to keep intermediate predator density relatively low. When intermediate predator density is at 
such low values, prey are released from predation pressure sufficiently to maintain non-zero population abundance.

Bistability arises in numerous models of ecosystem dynamics. For example, in a tri-trophic chemostat model with few patches, Suzuki and Yoshida (2012) found that dispersal of nutrients generates bistability. Similarly, Goldwyn and Hastings (2011) found multistability in a many-patch system. Furthermore, when patches are fewer, extinction becomes much more likely; Wilson et al. (1998) found a strong effect of lattice size whereby extinctions were more likely for models of smaller areas. In his tri-trophic metapopulation model, Jansen (1994) found results similar to ours in that top predator dispersal can both destabilize dynamics and generate bistability. In his model formulation, dispersal is non-instantaneous, generating a time lag in predator reactions to prey density. Dispersal is instantaneous and space is implicit in our model, however, and so the finding of bistability is apparently quite general. Although bistability can arise in one-dimensional models (e.g., Drury 2007) and two-dimensional models (e.g., Drury and Lodge 2009) that include a type III functional response, we are not aware of one- or two-dimensional models with a type II functional response, such as the two-dimensional Rosenzweig-MacArthur model has, exhibiting bistability.

These results do not imply that tri-trophic interactions always undermine the benefits of increased immigration among subpopulations, but they do suggest the need for awareness of the possibility of destabilization. For example, at high levels of immigration in each population, the dynamics of the model are stabilized. Destabilization only occurs at intermediate values of migration. Migration between subpopulations is common (e.g., cougars (Cooley et al. 2009)) and has been shown to contribute to global persistence, for example through the rescue effect, and distribution of risk arising from natural disasters. These mechanisms of persistence are thought to be especially relevant in the case of top predators because such predators are typically large-bodied, relatively rare, have sparser populations, and are wide-ranging with large habitat requirements (Wielgus 2002).

Our results suggest the need for further modeling studies, however. Specifically, there appears to be a need to study the dynamical consequences of both emigration and immigration in local populations with a coupled patch model. Jansen (1994) used Lotka-Volterra-style interactions in a three-species metapopulation model and found that migration of upper trophic levels can destabilize interactions with trophic levels below them. Those results, in conjunction with ours, suggest that there are complexities that arise in analyses of food chains at the landscape level. Nevertheless, organismal behavior is often more complex than that which our models portray. For example, in our model, top predator migration is constant, while in reality, migration is likely to vary with food availability, which in turn can be related to top predator density itself. Thus, including more complex behavioral mechanisms may erode the possibility of bistability.

We conclude that while migration between patches can increase the fraction of patches occupied, at intermediate levels, it can cause local populations to visit the small sizes in the troughs of cycles at which they are subject to local extinction through demographic and environmental stochasticity (Sole and Montoya 2006). Immigration therefore presents a dilemma for management of top predator populations, e.g., lynx reintroductions that rely on adequate migration from surrounding source populations.

In conclusion, we note that exhaustive bifurcation analysis of this model, considering $\mu_{x}, \mu_{y}$, and $\mu_{z}$, is a significant task that still remains to be completed. Future work could consider bifurcations resulting from varying these three parameters in a more comprehensive manner. In contrast, our study has focused on a few bifurcations selected for their relevance to the well-known and widely adopted conservation strategy of increasing immigration among top predators. Our results show that this strategy can backfire, which should be considered a possible outcome of actions aimed at improving immigration corridors for top predators.

Acknowledgments We thank Erwin Suscipto, Robert D. Holt, and an anonymous reviewer who made valuable comments that improved the clarity of the manuscript. We also wish to thank the developers of MATCONT. This work was supported by NSF award EF-0914347.

\section{Appendix A}

To non-dimensionalize the model, we begin by relegating all the units of our state variables to constants thus transforming the uppercase state variables into constants multiplied by unitless lowercase state variables (e.g., Brown 1991). Thus, the uppercase state variables of the model become,

$T \equiv c_{t} t, \quad X \equiv c_{x} x, \quad Y \equiv c_{y} y \quad Z \equiv c_{z} z$

Substituting these expressions for the uppercase variables into the model yields,

$\frac{c_{x}}{c_{t}} \frac{d x}{d t}=R c_{x} x\left(1-\frac{c_{x} x}{K}\right)-\frac{A_{x} c_{x} x c_{y} y}{B_{x}+c_{x} x}$,

$\frac{c_{y}}{c_{t}} \frac{d y}{d t}=\frac{C_{x, y} A_{x} c_{x} x c_{y} y}{B_{x}+c_{x} x}-d_{y} c_{y} y-\frac{A_{y} c_{y} y c_{z} z}{B_{y}+c_{y} y}$,

$\frac{c_{z}}{c_{t}} \frac{d z}{d t}=\frac{C_{y, z} A_{y} c_{y} y c_{z} z}{B_{y}+c_{y} y}-d_{z} c_{z} z$. 
We can immediately simplify these equations by dividing through each equation by $c_{x}, c_{y}$, and $c_{z}$, respectively, and multiplying each by $c_{t}$, which yields,

$$
\begin{aligned}
& \frac{d x}{d t}=R c_{t} x\left(1-\frac{c_{x} x}{K}\right)-\frac{c_{t} A_{x} x c_{y} y}{B_{x}+c_{x} x}, \\
& \frac{d y}{d t}=\frac{C_{x, y} c_{t} A_{x} c_{x} x y}{B_{x}+c_{x} x}-c_{t} d_{y} y-\frac{c_{t} A_{y} y c_{z} z}{B_{y}+c_{y} y}, \\
& \frac{d z}{d t}=\frac{C_{y, z} c_{t} A_{y} c_{y} y z}{B_{y}+c_{y} y}-c_{t} d_{z} z .
\end{aligned}
$$

We next factor $B_{x}$ and $B_{y}$ from the denominators of the functional responses in Eqs. 26-28 and group the parameters yielding,

$$
\begin{aligned}
& \frac{d x}{d t}=R c_{t} x\left(1-\frac{c_{x} x}{K}\right)-\frac{c_{t} A_{x} c_{y}}{B_{x}} \frac{x y}{\left(1+\frac{c_{x}}{B_{x}} x\right)} \\
& \frac{d y}{d t}=\frac{C_{x, y} c_{t} c_{x} A_{x}}{B_{x}} \frac{x y}{\left(1+\frac{c_{x}}{B_{x}} x\right)}-c_{t} d_{y} y-\frac{c_{t} A_{y} c_{z}}{B_{y}} \frac{y z}{\left(1+\frac{c_{y}}{B_{y}} y\right)} \\
& \frac{d z}{d t}=\frac{C_{y, z} c_{t} c_{y} A_{y}}{B_{y}} \frac{y z}{\left(1+\frac{c_{y}}{B_{y}} y\right)}-c_{t} d_{z} z
\end{aligned}
$$

We are now in a position to remove certain parameter groups. According to the pi theorem (Brown 1991), with $p$ as the number of original parameters and $u$ as the number of fundamental units, we need only retain $p-$ $u$ parameters, the remaining ones being set equal to 1 . Furthermore, we are free to rename the remaining parameter groups with single parameters, thus simplifying the equations.

Equations 3-5 had ten original parameters. There are four fundamental units including time, abundance of $X$, abundance of $Y$, and abundance of $Z$. Thus, we must retain six parameter groups to represent these units. We know that we wish to remove $R$ and $K$ and so we set,

$R c_{t} \equiv 1, \quad$ and $\quad \frac{K}{c_{x}} \equiv 1$,

which means that $c_{t}=\frac{1}{R}$ and $c_{x}=K$.

Similarly, because they are equal in the conventional form of the model, we set the coefficient of the related functional response to be equal. Specifically,

$\frac{c_{t} A_{x} c_{y}}{B_{x}}=\frac{C_{x, y} c_{t} c_{x} A_{x}}{B_{x}} \quad$ and $\quad \frac{c_{t} A_{y} c_{z}}{B_{y}}=\frac{C_{y, z} c_{t} c_{y} A_{y}}{B_{y}}$,

and solve these for $c_{y}$ and $c_{z}$, yielding,
Table 5 Renaming of nondimensional parameter groups to arrive at Eqs. 43-45, the conventional representation of the three-dimensional Rosenzweig-MacArthur model

\begin{tabular}{ll}
\hline Parameter group & New parameter \\
\hline$\frac{A_{x} C_{x, y} K}{R B_{x}}$ & $\alpha_{x}$ \\
$\frac{A_{y} C_{y, z} C_{x, y} K}{R B_{y}}$ & $\alpha_{y}$ \\
$\frac{c_{x}}{B_{x}}$ & $\beta_{x}$ \\
$\frac{c_{y}}{B_{y}}$ & $\beta_{y}$ \\
$\frac{d_{y}}{R}$ & $\delta_{y}$ \\
$\frac{d_{z}}{R}$ & $\delta_{z}$ \\
\hline
\end{tabular}

and

$$
\begin{aligned}
\frac{C_{t} A_{y} c_{z}}{B / y} & =\frac{C_{y, z} \mathscr{A t} c_{y} A_{y}}{B y}, \\
c_{z} & =C_{y, z} c_{y}, \\
& =C_{y, z} C_{x, y} K .
\end{aligned}
$$

Making these substitutions for $c_{t}, c_{x}, c_{y}$, and $c_{z}$ in the model yields,

$$
\begin{aligned}
& \frac{d x}{d t}=R c_{t} x\left(1-\frac{c_{x} x}{K}\right)-\frac{A_{x} C_{x, y} K}{R B_{x}} \frac{x y}{\left(1+\frac{c_{x}}{B_{x}} x\right)} \\
& \frac{d y}{d t}=\frac{C_{x, y} K A_{x}}{R B_{x}} \frac{x y}{\left(1+\frac{c_{x}}{B_{x}} x\right)}-\frac{d_{y}}{R} y-\frac{A_{y} C_{y, z} C_{x, y} K}{R B_{y}} \frac{y z}{\left(1+\frac{c_{y}}{B_{y}} y\right)} \\
& \frac{d z}{d t}=\frac{C_{y, z} C_{x, y} K c A_{y}}{R B_{y}} \frac{y z}{\left(1+\frac{c_{y}}{B_{y}} y\right)}-c_{t} d_{z} z
\end{aligned}
$$

We are now free to rename the remaining parameter groups with single Greek letters, with subscripts as needed,

With these parameter names, we have arrived at the conventional, non-dimensional form of the three-dimensional Rosenzweig-MacArthur model,

$$
\begin{aligned}
& \dot{x}=x(1-x)-\frac{\alpha_{x} x y}{1+\beta_{x} x}, \\
& \dot{y}=\frac{\alpha_{x} x y}{1+\beta_{x} x}-\delta_{y} y-\frac{\alpha_{y} y z}{1+\beta_{y} y}, \\
& \dot{z}=\frac{\alpha_{y} y z}{1+\beta_{y} y}-\delta_{z} z
\end{aligned}
$$


where $\dot{x} \equiv \frac{d x}{d t}, x$ represents prey population density, $y$ represents predator population density, $z$ represents top predator population density, the $\alpha_{i}$ represent scaled maximal attack rates, the $\beta_{i}$ represent the inverse of the prey density at which predators become half-saturated $(i \in\{x, y\})$, and the $\delta_{j}$ represent scaled non-predation mortality $(j \in\{y, z\})$.

\section{References}

Abdllaoui AE, Auger P, Kooi BW, de la Parra RB, Mchich R (2007) Effects of density-dependent migrations on stability of a two-patch predator-prey model. Math Biosci 210:335-354

Amarasekare P (2008) Spatial dynamics of foodwebs. Ann Rev Ecol Evol Syst 39:479-500

Bascompte J, Solé RV (1996) Habitat fragmentation and extinction thresholds in spatially explicit models. J Anim Ecol 65:465-473

Beschta RL (2003) Cottonwoods, elk, and wolves in the Lamar valley of yellowstone national park. Ecol Appl 13(5):1295-1309

Blasius B, Huppert A, Stone L (1999) Complex dynamics and phase synchronization in spatially extended ecological systems. Nature 399:354-359

Boer MP, Kooi BW, Kooijman SALM (2001) Multiple attractors and boundary crises in a tri-trophic food chain. Math Biosci 169:109128

Brown RA (1991) Fluid mechanics of the atmosphere. Academic Press Inc, New York

Cooley HS, Wielgus RB, Koehler GM, Robinson HS, Maletzke BT (2009) Source populations in carnivore management: cougar demography and emigration in a lightly hunted population. Anim Conserv 12:321-328

Diamond JM (1984). In: M.H. Nitecki (ed) "Normal" extinctions of isolated populations. Chicago University Press, pp 191-246 in Extinctions

Doebeli M (1995) Dispersal and dynamics. Theor Popul Biol 47:82106

Drury KLS (2007) Shot noise perturbations and mean first passage times between stable states. Theor Popul Biol 72:153-166

Drury KLS, Lodge DM (2009) Using mean first passage times to quantify equilibrium resilience in perturbed intraguild predation systems. Theor Ecol 2:41-51

Goldwyn EE, Hastings A (2011) The roles of the Moran effect and dispersal in synchronizing oscillating populations. J Theor Biol 289:237-246

Govaerts W, Kuznetsov Yu. A, De Witte V, Dhooge A., Meijer HGE, Mestrom W, Riet AM, Sautois B (2011) MATCONT and CL_MATCONT: Continuation toolboxes in MATLAB

Hanski I (1994) A practical model of metapopulation dynamics. J Anim Ecol 63:151-162

Hastings A (1996). In: Polis GA, Winemiller KO (eds) Food webs. Integration of patterns and dynamics. Chapman \&amp; Hall, New York

Hastings A, Powell T (1991) Chaos in a three-species food chain. Ecology 72(3):896-903

Heithaus MR, Frid A, Wirsing AJ, Dill LM, Fourqurean JW, Burkholder D, Thomson J, Bejder L (2007) State-dependent risktaking by green sea turtles mediates top-down effects of tiger shark intimidation in a marine ecosystem. J Anim Ecol 76(5):837-844

Hill JK, Thomas CD, Lewis OT (1996) Effects of habitat patch size and isolation on dispersal by Hesperia comma butterflies: implications for metapopulation structure. J Anim Ecol 65:725735
Holt RD, Freedman HI, Strobek C (1983) Models for peripheral populations: the role of immigration. in Population Biology Lecture notes in biomathematics, number 52. Springer-Verlag, Berlin

Holt RD (1985) Population dynamics in two-patch environments: some anomalous consequences of an optimal habitat distribution. Theor Popul Biol 28:181-208

Holt RD (1993) Ecology at the mesoscale: the influence of regional processes on local communities. Species diversity in ecological communities 101:77-88

Holt RD (2002) Food webs in space: on the interplay of dynamic instability and spatial processes. Ecol Res 17:261-273

Holyoak M, Lawler S (1996) Persistence of an extinction-prone predator-prey interaction through metapopulation dynamics. Ecology $77: 1867-1879$

Jansen VAA (1994) Effects of dispersal in a tri-trophic metapopulation model. J Math Biol 34:195-224

Klebanoff A, Hastings A (1994) Chaos in three species food chains. J Math Biol 32:427-451

Koehler GM, Maletzke BT, von Kienast JA, Aubry KB, Wielgus RB, Naney R. H (2008) Habitat fragmentation and the persistence of lynx populations in Washington State. J Wildl Manag 72(7):15181524

Krebs CJ, Boutin S, Boonstra R, Sinclair ARE, Smith JNM, Dale MRT, Martin K, Turkington R (1995) Impact of food and predation on the snowshoe hare cycle. Science 269:1112-1115

Kuang Y, Takeuchi Y (1994) Predator-prey dynamics in models of prey dispersal in two-patch environments. Math Biosci 120:77-98

Kuznetsov YA (2004) Elements of applied bifurcation theory. Springer-Verlag, New York

Kuznetsov YA, Rinaldi S (1996) Remarks on food chain dynamics. Math Biosci 134:1-33

Kuznetsov YA, De Feo O, Rinaldi S (2001) Belyakov homoclinic bifurcations in a tritrophic food cha in model. SIAM J Appl Math 62(2):462-487

McCallum HI (1992) Effects of immigration on chaotic population dynamics. J Theor Biol 154:227-284

McCann K, Yodzis P (1996) Biological conditions for chaos in a threespecies food chain. Ecology 75:561-564

Miotto RA, Cervini M, Figueiredo MG, Begotti RA, Galetti PM (2011) Genetic diversity and population structure of pumas (Puma concolor) in southeastern Brazil: implications for conservation in a human-dominated landscape. Conserv Genet 12:14471455

Paine RT (1966) Food web complexity and species diversity. Am Nat 100(910):65-75

Ripple WJ, Beschta RL (2004) BioScience 8:755-766

Robinson HS, Wielgus RB, Cooley HS, Cooley SW (2008) Sink populations in carnivore management: cougar demography and immigration in a hunted population. Ecol Appl 18(4):10281037

Rodriguez A, Delibes M (2003) Population fragmentation and extinction in the Iberian lynx. Biol Conserv 109:321-331

Rosenzweig M (1971) The paradox of enrichment. Science 171:385387

Ruxton GD (1996) Chaos in a three-species food chain with a lower bound on the bottom population. Ecology 77(1):317-319

Sergio F, Newton I, Marchesi L, Pedrini P (2006) Ecologically justified charisma: preservation of top predators delivers biodiversity conservation. J Appl Ecol 43:1049-1055

Solé RV, Montoya JM (2006). In: Pascual M, Dunne JA (eds) In Ecological networks: linking structure to dynamics in food webs. Oxford University Press, New York

Stone L (1993) Period-doubling reversals and chaos in simple ecological models. Nature 365:617-620 
Suzuki K, Yoshida T (2012) Non-random spatial coupling induces desynchronization, chaos and multistability, in a predator-preyresource system. J Theor Biol 300:81-90

Wielgus RB (2002) Minimum viable population and reserve sizes for naturally regulated grizzly bears in British Columbia. Biol Conserv 106:381-388
Wiens JA (1989) Spatial scaling in ecology. Funct Ecol 3:385-397

Wilson HB, Hassell MP, Holt RD (1998) Persistence and area effects in a stochastic tritrophic model. Am Na 151(6):587-595

van Voorn GAK, Kooi BW, Boer MP (2010) Ecological consequences of global bifurcations in some food chain models. Math Biosci 226:120-133 